\title{
CORRIGENDUM: ALMOST SURE RATES OF MIXING FOR I.I.D. UNIMODAL MAPS
}

\author{
By ViVIANE BALADI, MiCHAEL BENEDICKS AND \\ VÉRONIQUE MAUME-DESCHAMPS
}

\begin{abstract}
The definition of the return time (p. 117) and the beginning of the proof of Proposition 8.3 of our paper in Vol. 35 of Ann. Scient. Éc. Norm. Sup. (2002) are not correct. We give an amended version which shows that none of the statements are affected. We take this opportunity to correct some other mistakes (without consequences), e.g. in Sublemma 7.2(3) and Lemma 7.10.

Published by Éditions scientifiques et médicales Elsevier SAS
\end{abstract}

RÉSUMÉ. - La définition du temps de retour (p. 117) et le début de la preuve de la Proposition 8.3 dans notre article du Vol. 35 des Ann. Scient. Éc. Norm. Sup. (2002) sont incorrects. Nous donnons ici les arguments montrant que les résultats de notre article n'en sont pas affectés.

Published by Éditions scientifiques et médicales Elsevier SAS

The definition of the return time on p. 117 and the beginning of the proof of Proposition 8.3 on pp. 118-120 are not correct. Indeed the large image property claimed in the middle of p. 120 is not guaranteed by the construction, and the interplay between the hyperbolic-time partition in Section 7.3 and Lemma 7.10 must be monitored more carefully. The amended version is given below. None of the statements are affected by this.

(Note also that the right-hand-side of the bound in Sublemma 7.2(3) should be

$$
\max \left(\mathrm{e}^{-2 \eta k}, \varepsilon^{\eta}\right) \min \left(\mathrm{e}^{2 k}, \varepsilon^{-1}\right)
$$

As a consequence, Lemma 7.10 is only true for $k \leqslant(1 / 2) \log (1 / \varepsilon)$. This does not cause problems in Lemma 8.1: if $1-3 \eta \geqslant 1 / 2$, continue iterating, obtaining $1-5 \eta, 1-9 \eta$ etc. until $1-\ell \eta<1 / 2$.

V.B. thanks S. Gouëzel for pointing out the mistake and for very helpful discussions.

\section{Corrections in $\S 8$}

The definition of escape times on pp. 116-117 and Corollary 8.2 on p. 117 are not neeeded. Replace the text after the proof of Lemma 8.1 (p. 116) and before "Estimate (8.3) for $\widehat{R}_{\omega}$ " (p. 119) by:

Proposition 8.3 (Partition $\Lambda_{i}(\omega)$ and return times $R_{\omega}$ ). - There exist $\Omega_{1} \subset \Omega_{0}$ of full measure, and for all $\omega \in \Omega_{1}$, a countable (modulo zero measure sets) partition $\mathcal{Z}_{\omega}$ of $\Lambda$, into subintervals $\left\{\Lambda_{i}=\Lambda_{i}(\omega) \mid i \in \mathbb{Z}_{+}\right\}$and a return-time function $R_{\omega}$, defined almost everywhere on $\Lambda$, and satisfying Axioms (A.I)-(A.IV) from Section 3.2. 
Additionally, there are a random variable $n_{1}(\omega)$ and constants $C(\varepsilon) \geqslant 1, C_{1}(\varepsilon)>C_{2}(\varepsilon)>1$ so that for all $\ell \geqslant n_{1}(\omega)$

$$
\begin{gathered}
\operatorname{Leb}\left(\left\{x \in \Lambda \mid R_{\omega}(x)>\ell\right\}\right)<C(\varepsilon) \mathrm{e}^{-\left(\ell^{1 / 4} / C_{1}(\varepsilon)\right),} \\
P\left(\left\{\omega \in \Omega_{1} \mid n_{1}(\omega)>\ell\right\}\right)<C(\varepsilon) \mathrm{e}^{-\left(\ell^{1 / 4} / C_{2}(\varepsilon)\right) .}
\end{gathered}
$$

We may replace the right-hand-sides in both inequalities by $C(\varepsilon) \mathrm{e}^{-\ell^{u}}$ for $0<u<1 / 4$.

Proof of Proposition 8.3. - We first define a partition $\widehat{\Lambda}_{i}(\omega)$ and estimate return times $\widehat{R}_{\omega}(x)$ corresponding to the first moment when one of the $\Lambda_{ \pm}$is exactly covered (with expansion and distorsion control).

Fix $\omega \in \Omega$. Our starting point is the countable decomposition from Section 7.3:

$$
\Lambda_{+} \cup \Lambda_{-}=\bigcup_{m=p_{0}}^{\infty} \bigcup_{J \in \mathcal{R}_{m}(\omega)} J,
$$

where we have taken $p_{0} \geqslant C \log (1 / \varepsilon)$ (the bound on $t(J)$ from Lemma 8.1) and we have introduced the intervals $\Lambda_{ \pm}$and $\widetilde{\Lambda}_{ \pm} \backslash \Lambda_{ \pm}$in the initial partition $\mathcal{Q}$. For (almost) each $x \in \Lambda_{+} \cup \Lambda_{-}$, there is $J \in \mathcal{R}_{m}(\omega)$ containing $x$. We shall further partition it (if needed). By construction, almost every initial $x$ will be contained in an interval $\widehat{J}$ of the subpartition, and Lemma $7.9(2,3)$ will hold (injectively) for $\widehat{J}$ and an iterate $\hat{m}(\widehat{J})<\infty$. Additionally, the Markov property $f^{\hat{m}}(\widehat{J})=\Lambda_{+}$or $f^{\hat{m}}(\widehat{J})=\Lambda_{-}$will hold. The partition $\widehat{\Lambda}_{i}$ will be the partition into intervals $\widehat{J}$, and the return time on $\widehat{J}$ will be $\widehat{R}_{\omega} \equiv \hat{m}(\widehat{J})$.

To control the return-time asymptotics, we shall introduce a sequence of stopping times

$$
\widehat{T}_{i}=\widehat{T}_{\omega, i}: \Lambda_{+} \cup \Lambda_{-} \rightarrow \mathbb{N} \cup\{\infty\}
$$

with ... (no more changes on p. 118 until after (8.5), except that Lemma 7.8 should be mentioned instead of Corollary 8.2).

Let us now define the stopping times, using again the notation from Lemma 8.1. We say that $\widehat{T}_{\omega, 1}$ is defined at $x \in \Lambda_{+} \cup \Lambda_{-}$if there is $J_{1} \in \mathcal{R}_{m_{1}}(\omega)$ with $x \in J_{1}$ (hence, $f^{m_{1}+t\left(J_{1}\right)}\left(J_{1}\right)$ contains $f^{m_{1}+t\left(J_{1}\right)}(x)$ and covers $\widetilde{\Lambda}_{+}$or $\left.\widetilde{\Lambda}_{-}\right)$. We then set

$$
\widehat{J_{1}}=\left(f_{\omega}^{m_{1}}\right)^{-1} \circ\left(\left.f_{\sigma^{m_{1}} \omega}^{t\left(J_{1}\right)}\right|_{\tilde{J}_{1}}\right)^{-1}\left(\Lambda_{+}\right) \quad \text { or } \quad \Lambda_{-}
$$

and

$$
\widehat{T}_{\omega, 1}(x)= \begin{cases}m_{1}+t\left(J_{1}\right)=\widehat{R}_{\omega}(x) & \text { if } x \in \widehat{J}_{1}, \\ m_{1} & \text { otherwise. }\end{cases}
$$

The leftover interval(s) $J_{1} \backslash \widehat{J}_{1}$, correspond to points which have reached a stopping time but not returned yet. They will be further partitioned. For this, let

$$
\Theta_{\omega, 1}=\left\{x \in \Lambda_{+} \cup \Lambda_{-} \mid \widehat{T}_{\omega, 1}(x) \text { is defined }\right\} .
$$

For $x \in \Theta_{\omega, 1} \backslash\left\{\widehat{R}_{\omega}(x)=\widehat{T}_{\omega, 1}(x)\right\}$, we say that $\widehat{T}_{\omega, 2}$ is defined at $x$ if there are $m_{2}>p_{0}$ and $J_{2} \in \mathcal{R}_{m_{2}}\left(\sigma^{\widehat{T}_{\omega, 1}(x)} \omega, L_{1}(x)\right)$ with $f_{\omega}^{\widehat{T}_{\omega, 1}(x)}(x) \in J_{2}$. Here, the intervals in $\mathcal{R}_{m_{2}}\left(\sigma^{\widehat{T}_{\omega, 1}(x)} \omega, L_{1}\right)$ are obtained by applying the partitioning rules in Section 7.3 to the interval in $L_{1}=L_{1}(x)=$ $f_{\omega}^{m_{1}}\left(J_{1} \backslash \widehat{J}_{1}\right)$ containing $f_{\omega}^{m_{1}}(x)$. We describe this new mechanism in detail next, noting first 
that it may produce a different partition than the intersection of $L_{1}$ with the partition described in Section 7.3, but that Lemma 8.1 will hold for the modified partition (because a modified version of Lemma 7.9 will hold).

More precisely, when applying the mechanism in Section 7.3 to a subinterval $L^{\prime} \subset I$ (for example $L^{\prime}=L_{1}(x)$ ) (P.I) should be replaced by:

$(\widehat{P} . \mathrm{I}): H_{m}^{*}\left(\omega^{\prime}\right) \cap L^{\prime} \subset \cup_{j=p_{0}}^{m} \bigcup_{J \in \mathcal{R}_{j}\left(\omega^{\prime}, L^{\prime}\right)} J$, and $J \cap H_{m}^{*}\left(\omega^{\prime}\right) \neq \emptyset$ for all $J \in \mathcal{R}_{m}\left(\omega^{\prime}, L^{\prime}\right)$ and $m \geqslant p_{0}$.

Writing $\mathcal{S}_{m}\left(\sigma^{m_{1}} \omega, L_{1}\right)$ for $L_{1} \backslash \bigcup_{j=p_{0}}^{m} \bigcup_{J \in \mathcal{R}_{j}\left(\sigma^{m_{1}} \omega, L_{1}\right)} J$, Lemma 7.8 then implies that

$$
\operatorname{Leb}\left(\mathcal{S}_{m}\left(\sigma^{m_{1}} \omega, L_{1}\right)\right) \leqslant C \mathrm{e}^{-\zeta(\varepsilon) m}
$$

for all $m \geqslant n_{0}\left(\sigma^{m_{1}} \omega\right)$.

(P.II) is unchanged and will hold by construction, guaranteeing the required bounded distorsion. (P.IV) is also unchanged.

The large image property (P.III) on p. 112 (essential in the proof of Lemma 7.9(1)) must be replaced by the following modified large image property:

( $\widehat{\mathrm{P}}$.III): for each $J \in \mathcal{R}_{m}(\omega, L)$ either (P.III) holds or there is $0 \leqslant j \leqslant C \log (1 / \varepsilon)$ so that $f_{\omega}^{j}(J)$ contains an $\hat{I}_{k, \ell}$. (See the proof of Lemma 7.10, pp. 115-116, noting that $(-\delta, \delta)$ contains infinitely many intervals $I_{k}$. In fact, it is useful to replace the three adjacent $\hat{I}_{k, \ell} \mathrm{s}$ in (7.21), (7.24), but not (7.25), by five such adjacent intervals.) Then, changing slightly the computations on pp. 113-114 to accommodate for the $k^{-2}$ factor in the intervals $\hat{I}_{k, \ell}$ (note that we can assume $\left.\mathrm{e}^{-k} \geqslant \sqrt{\varepsilon} \mathrm{e}^{-r_{j}(\omega, x)+1}\right)$, we get $\left|f_{\omega}^{m}(J)\right| \geqslant \varepsilon^{1-2 \eta} /\left(C \log \left(\varepsilon^{2 \eta-1}\right)\right)$ almost as in Lemma 7.9(2), and enough for the purposes of the proof of Lemma 8.1.

Let

$$
\widehat{J_{2}}=\left(f_{\omega}^{\widehat{T}_{\omega, 1}(x)+m_{2}}\right)^{-1} \circ\left(\left.f_{\sigma^{m} \omega_{\omega}}^{t\left(J_{2}\right)}\right|_{\tilde{J}_{2}}\right)^{-1}\left(\Lambda_{ \pm}\right) .
$$

We set $\widehat{T}_{\omega, 2}(x)$ to be

$$
\widehat{R}_{\omega}(x)=\widehat{T}_{\omega, 1}(x)+m_{2}+t\left(J_{2}\right)
$$

if $f_{\omega}^{\widehat{T}_{\omega, 1}(x)}(x) \in \widehat{J}_{2}$, and otherwise $\widehat{T}_{\omega, 2}(x)=\widehat{T}_{\omega, 1}(x)+m_{2}$. For general $k \geqslant 2$, we let

$$
\Theta_{\omega, k}=\left\{x \mid \widehat{T}_{\omega, k}(x) \text { is defined }\right\},
$$

and we define $\widehat{T}_{\omega, k+1}$ on $\Theta_{\omega, k+1} \subset \Theta_{\omega, k} \backslash\left\{\widehat{R}_{\omega}(x)=\widehat{T}_{\omega, k}(x)\right\}$ if there is $m_{k+1} \geqslant p_{0}$ and $J_{k+1} \in \mathcal{R}_{m_{k+1}}\left(\sigma^{\widehat{T}_{\omega, k}(x)} \omega, L_{k}\right)$ with $f_{\omega}^{\widehat{T}_{\omega, k}(x)}(x) \in J_{k+1}$. The relation (8.1) (and thus (8.2)) is an immediate consequence of the definition.

The modifications in the rest of the proof of Proposition 8.3 are:

(1) Before the proof of estimate (8.3) on p. 119, insert: "Note that the total distorsion may be estimated by $C(\varepsilon)^{i} \xi^{\ell}$, where the return time $\ell$ is at least $p_{0}$ times the number $i$ of stopping times and $\beta<\xi<1$ (if necessary, take a larger $p_{0}(\varepsilon)$ to ensure $C(\varepsilon)^{1 / p_{0}} \xi<1$ )".

(2) In the proof of estimate (8.3) on p. 119: replace " $J$ is an interval of $\mathcal{R}_{m}\left(\sigma^{n^{\prime}} \omega\right)$ " by " $J$ is an interval of $\mathcal{R}_{m}\left(\sigma^{n^{\prime}} \omega, L\right)$ ”.

(3) On p. 120, replace the "absolute bounds from Corollary 8.2 " by "( $\widehat{P} .1)$ " (twice).

(4) On p. 120, replace "large image properties from Lemma 8.1" by "the modified large image property above".

(5) Replace the notation $R_{\omega}^{*}$ by $R_{\omega}$ on pp. 121-122. 


\section{The rest of the paper}

In Section 9, (A.IV) now comes from Proposition 8.3 (instead of Lemmas 7.9-7.10).

We take this opportunity to mention typographical mistakes in Section 7.3: in the first line of p. 112: $H_{k}(\omega) \subset \bigcup_{J \in \mathcal{R}_{k}(\omega)} J$ should be replaced by $H_{k}(\omega) \subset \bigcup_{j=p_{0}}^{k} \bigcup_{J \in \mathcal{R}_{j}(\omega)} J$; in the second line $n \geqslant n_{0}(\omega)$ must be replaced by $m \geqslant n_{0}(\omega)$.

(Manuscrit reçu le 2 juillet 2002; accepté, après révision, le 8 novembre 2002.)

Viviane BALADI I.H.É.S.

35 , route de Chartres

F-91440 Bures-sur-Yvette, France

Current address: C.N.R.S.,

Institut Mathématique de Jussieu,

4, place Jussieu

F-75251 Paris, France

E-mail: baladi@math.jussieu.fr

Michael BENEDICKS

Royal Institute of Technology,

S-100 44 Stockholm, Sweden

E-mail: michaelb@math.kth.se

Véronique MaUme-Deschamps

Laboratoire de Topologie,

Université de Bourgogne,

F-21078 Dijon, France

E-mail: vmaume@u-bourgogne.fr 